\title{
Analytical chemistry and the Chemical Weapons Convention
}

\author{
Marc-Michael Blum • R. V. S. Murty Mamidanna
}

Published online: 14 June 2014

(C) Springer-Verlag Berlin Heidelberg 2014

The entry into force of the Chemical Weapons Convention (CWC) on the 29th of April 1997 was a landmark success in the field of arms control and disarmament. Fig. 1 The CWC outlaws the use, production and stockpiling of chemical weapons and bans or regulates precursors. A signature element of the CWC is the complex and far-reaching verification mechanism that is implemented by the Technical Secretariat of the Organisation for the Prohibitions for Chemical Weapons (OPCW) located in The Hague in the Netherlands. This includes declaration requirements for existing stockpiles, precursors and production facilities and their subsequent destruction under constant supervision of OPCW inspectors. Additional requirements include declaration of certain industrial sites and activities, inspections of industry sites as confidence-building measures and the requirement for state parties to the $\mathrm{CWC}$ to implement national legislation. In the case of a suspected violation of the CWC, the policy-making organs of the OPCW can invoke several mechanisms, including challenge inspections or investigations of alleged use. The full text of the CWC, the schedules of chemicals and the verification annex as well as detailed information on the organisational structure of the OPCW and its latest activities can be accessed on the website at http://www.opcw.org.

Published in the topical collection Analysis of Chemicals Relevant to the Chemical Weapons Convention with guest editors Marc-Michael Blum and R. V. S. Murty Mamidanna.

M.-M. Blum $(\bowtie) \cdot$ R. V. S. M. Mamidanna

OPCW Laboratory, Organisation for the Prohibition of Chemical

Weapons, Heulweg 28-30, 2288 GN Rijswijk, The Netherlands

e-mail: marc.blum@opcw.org
Although a number of verification activities, especially those in the chemical industry, centre on checking submitted declarations with records available on-site, the only way to gather factual evidence on the presence (or absence) of chemicals relevant to the CWC is by the use of techniques from the toolkit offered by analytical chemistry. Analysis can be quantitative in nature, for example to ensure that residual agent concentrations in the waste stream at destruction facilities are below the allowed threshold, but in most other scenarios analysis is predominantly qualitative - a relevant chemical is either present or not present. Every year a number of industry inspections are conducted with a sampling and analysis component. This is a confidence-building measure and is conducted in an on-site laboratory by analytical chemist inspectors using OPCW equipment. The analytical workhorse of these missions is a standard gas chromatography (GC)-mass spectrometry (MS) instrument. Mission instruments are maintained and certified by the OPCW laboratory under an ISO 17025 accredited quality system.

The verification annex of the $\mathrm{CWC}$ also contains provisions to analyse samples off-site. This is of special importance in the case of politically sensitive missions such as challenge inspections or investigations of alleged use. For off-site analysis, the OPCW relies on an international network of partner laboratories (currently 21) designated by the Director General of the OPCW. To maintain their designation, these laboratories must have an accredited quality system and they must successfully participate in proficiency tests organised by the OPCW Laboratory (accredited under ISO 17043) at least once a year. In their last three tests, the laboratories must re- 
ceive grades of either A,A,A or A,A,B, where an A grade is awarded to laboratories that identified all spiking chemicals in the test and have no reporting errors, and a B grade indicates either a missed spiking chemical or a reporting error that leads to a non-scoring chemical. Laboratories with more than one B grade or even lower grades are suspended and cannot receive authentic samples for analysis. Also, the test scheme has zero tolerance for false positives. Reporting a false positive in a proficiency test results in failure of the test, and the laboratory loses its designation status. The fact that the number of possible reportable chemicals is unlimited (e.g. Schedule 2.B.04 in the annex on chemicals of the CWC contains an infinite number of chemicals) and that some of the spiking chemicals in proficiency tests are often not found in any spectral databases, together with the strict performance requirements outlined above, demonstrates the excellent capabilities of the laboratories. Even though the analytical technique of choice in the early days of the OPCW proficiency testing programme was GC-MS, this has changed over the past 35 tests. Today, techniques include GC-flame photometric detection/nitrogen-phosphorus detection/atomic emission detection, GC-MS (electron ionization and chemical ionization), GC-MS/ MS, liquid chromatography (LC)-MS, LC-MS/MS, GC-high-resolution MS, LC-high-resolution MS, NMR spectroscopy and Fourier transform IR spectroscopy, to name just the most prominent ones. Combined with (micro)synthetic capabilities, this allows structural elucidation of even those chemicals not found in available databases, which nevertheless remain an important source for reference data. In addition to data available from commercial sources, the OPCW also maintains its own database of chemicals scheduled under the CWC. The OPCW Central Analytical Database is available to all member states, and currently contains about 5,200 MS spectra, 4,500 retention indices, 1,400 NMR spectra and 1,000 IR spectra.

Sampling and analysis of environmental samples can reveal the presence or absence of chemical agents (and/or their degradation products), but in order to assess if a potential victim was exposed, the analysis of biomedical samples is required. Blood and urine samples are preferred as they are easily collected. Analytical procedures in this area have advanced significantly over the past few years. Apart from the analysis of free agents and their metabolites, adducts of chemical warfare agents with biomolecules such as proteins and DNA are of main interest as they allow retrospective identification of exposure for much longer times owing to their extended persistence in the human body. To enhance the capabilities available to the OPCW and to move towards a possible specific designation of laboratories for biomedical sample analysis, the OPCW has conducted a number of exercises over the past few years. The fourth exercise was concluded in spring 2014, and tasked participants with the analysis of intact adducts of organophosphorus nerve agents with proteins such as serum albumin and butyrylcholinesterase.

The analytical techniques and procedures covered in this topical collection are as diverse as the analytical challenges encountered in the field. Chemically diverse analytes are encountered in a variety of matrices often with a significant number of unknown interferences, requiring advanced separation techniques and powerful data deconvolution solutions. Low concentrations of analytically relevant species in biomedical samples can require trace analysis in the sub-parts-per-billion region, and there is a constant search for new relevant biomarkers.

Recent events have shown that the use of chemical weapons is still a real possibility and present danger. Analytical chemistry has come a long way in its support of a universal and global ban on these weapons, and we hope that the readers of this topical collection will gain new insights into a field that practically contributes to global arms control and disarmament.

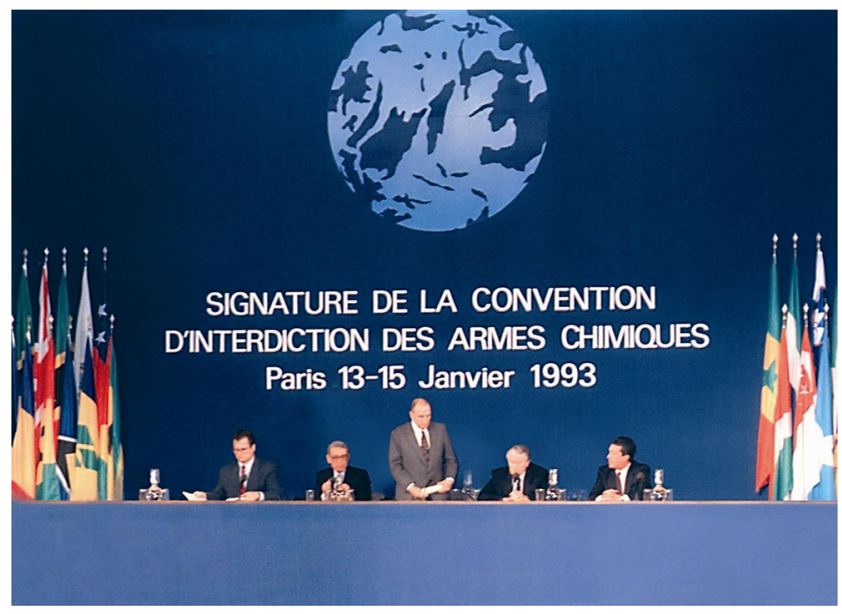

Fig. 1 The Chemical Weapons Convention (CWC) opened for signature on 13 January 1993 and entered into force on 29 April 1997. Today, 190 countries have signed and ratified the CWC. (Photograph from the Organisation for the Prohibition of Chemical Weapons) 


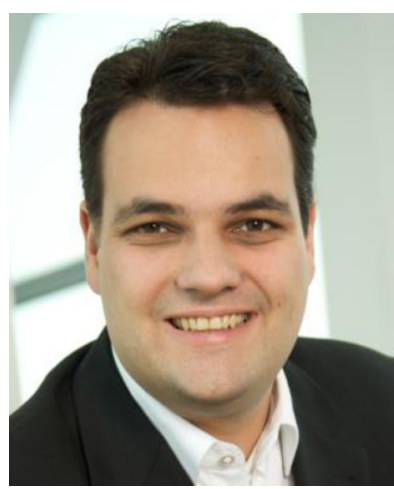

Marc-Michael Blum has been a senior analytical chemist in the Laboratory of the Organisation for the Prohibition of Chemical Weapons (OPCW) in Rijswijk, the Netherlands, since 2012. He started work in the area of defence against chemical weapons as a $\mathrm{Ph}$.D. student at the University of Frankfurt in 2003, exploring structural and mechanistic aspects of a nerve-agent-detoxifying enzyme. He further explored the field of enzymatic decontamination as an independent researcher, and developed an enzyme-based skin decontaminant in cooperation with the Bundeswehr Institute for Pharmacology and Toxicology in Munich. Prior to joining the OPCW, he spent 2 years as a Director's Fellow in the Bioscience Division at Los Alamos National Laboratory continuing to perform research in the field. Besides a Ph.D. degree in biochemistry (Johann Wolfgang Goethe-Universität Frankfurt am Main, Germany), he holds master degrees in chemistry (Technische Universität Braunschweig, Germany) and war studies (King's College London, UK). He is currently the analytical chemist responsible for conducting OPCW proficiency tests to designate laboratories for off-site analysis.

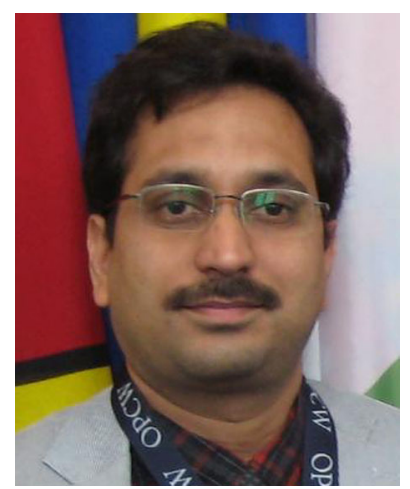

R.V.S. Murty Mamidanna is a senior analytical chemist in the Laboratory of the Organisation for the Prohibition of Chemical Weapons (OPCW) in Rijswijk, the Netherlands. Prior to joining the OPCW, he obtained his Ph.D. degree on mass spectral studies on Chemical Weapons Convention-related chemicals under the supervision of M. Vairamani at the National Centre for Mass Spectrometry, Indian Institute of Chemical Technology, Hyderabad, India, a laboratory designated by the OPCW. Later, he worked with Edwin de Pauw and Valerie Gabelica's research group as a postdoctoral researcher on the Alzheimer's Disease Centennial Project at the University of Liege, Belgium, and studied non-covalent protein-drug interactions using mass spectrometry. Presently, he is responsible for on-site sampling and analysis missions and biomedical confidence-building exercises conducted by the OPCW. His research interests include mass spectrometry, chromatography and organic synthesis. 